\title{
Integrated Multimodal Interaction Using Texture Representations ${ }^{\underline{4}}$
}

\author{
Auston Sterling, Ming C. Lin \\ University of North Carolina at Chapel Hill
}

\begin{abstract}
In this paper, we explore texture mapping as a unified representation for enabling realistic multimodal interaction with finelydetailed surfaces. We show how both normal maps and relief maps can be adopted as unified representations to handle collisions with textured rigid body objects, synthesize complex sound effects from long lasting collisions and perform rendering of haptic textures. The resulting multimodal display system allows a user to see, hear, and feel complex interactions with textured surfaces. By using texture representations for seamlessly integrated multimodal interaction instead of complex triangular meshes otherwise required, this work is able to achieve up to 25 times performance speedup and reduce up to six orders of magnitude in memory storage. We further validate the results through user studies to demonstrate the effectiveness of texture representations for integrated multimodal display.
\end{abstract}

\section{Introduction}

2 In computer graphics, texture mapping has been one of the 3 most widely used techniques to improve the visual fidelity of ${ }_{4}$ objects while significantly accelerating the rendering performanc 5 There are several popular texture representations, such as dis${ }_{6}$ placement maps [1], bump mapping with normal maps [2, 3], 7 parallax maps [4, 5], relief maps [6, 7], etc., and they are used 8 mostly as "imposters" for rendering static scenes. These tex${ }_{9}$ tures are usually mapped onto objects' surfaces represented with 10 simplified geometry. The fine details of the objects are visually ${ }_{11}$ encoded in these texture representations. By replacing the ge12 ometric detail with a texture equivalent, the resulting rendered ${ }_{13}$ image can be made to appear much more complex than its un${ }_{14}$ derlying polygonal geometry would otherwise convey. These 15 representations also come with a significant increase in perfor${ }_{16}$ mance: texture maps can be used for real-time augmented and 17 virtual reality (AR/VR) applications on low-end commodity de18 vices.

19 Sensory conflict occurs when there is a mismatch between 20 information perceived through multiple senses and can cause 21 a break in immersion in a virtual environment. When textures 22 are used to represent complex objects with simpler geometry, 23 sensory conflict becomes a particular concern. In an immersive 24 virtual environment, a user may see a rough surface of varying 25 heights and slopes represented by its texture equivalent mapped ${ }_{26}$ to a flat surface. In the real world, objects behave very dif27 ferently when bouncing, sliding, or rolling on bumpy or rough 28 surfaces than they do on flat surfaces. With visually complex 29 detail and different, contrasting physical behavior due to the ${ }_{30}$ simple flat surface, sensory conflict can easily occur-breaking ${ }_{31}$ the sense of immersion in the virtual environment. In order to

\footnotetext{
http://gamma.cs.unc.edu/MultiDispTexture
}

${ }_{32}$ capture such behaviors, the geometry used in a physics simu${ }_{3}$ lator would usually require a fine triangle mesh with sufficient 34 surface detail, but in most cases a sufficiently fine mesh is un35 available or would require prohibitive amounts of memory to 36 store.

37 Since the given texture maps contain information about the ${ }_{38}$ fine detail of the mapped surface, it is possible to use that infor39 mation to recreate the behavior of the fine mesh. Haptic display 40 and sound rendering of textured surfaces have both been inde${ }_{41}$ pendently explored [8, 9], but texture representations of detail 42 have not been previously used for visual simulation of dynamic ${ }_{43}$ behavior due to collisions and contacts between rigid bodies. ${ }_{44}$ For example, the system for sound rendering of contacts with 45 textured surfaces [9] displays a pen sliding smoothly across 46 highly bumpy surfaces. While the generated sound from this 47 interaction is dynamic and realistic, the smooth visual move48 ment of the pen noticeably does not match the texture implied 49 by the sound. In order to minimize sensory conflict, it is critical 50 to present a unified and seamlessly integrated multimodal dis51 play to users, ensuring that the sensory feedback is consistent 52 across the senses of sight, hearing, and touch for both coarse 53 and fine levels of detail.

$54 \quad$ Motivated by the need to address the sensory conflict due ${ }_{55}$ to the use of textures in a multimodal virtual environment, we ${ }_{56}$ previously examined the use of normal mapping as a unified 57 representation of fine detail for sight, hearing, and touch [10]. ${ }_{58}$ In this paper, we explore both normal maps and relief maps for 59 integrated multimodal display. The main results of this work 60 include:

- A new effective method for visual simulation of physical behaviors for rigid objects textured with normal maps;

- A seamlessly integrated multisensory interaction system using normal maps; 

${ }_{71}$ cuss why we have selected normal and relief maps as our tex72 ture representations for multimodal display. We then describe 73 how each mode of interaction can specifically use normal maps 74 to improve perception of complex geometry (Sec. 3). We high75 light the behavior of virtual objects as they interact with a large 76 textured surface, and describe a new method to improve vi77 sual perception of the simulated physical behaviors of colliding 78 objects with a textured surface using normal maps. We also 79 demonstrate how to use the same normal maps in haptic display 80 and sound rendering of textured surfaces. We describe how the ${ }_{81}$ additional depth information in relief maps can be used to im82 prove each mode of interaction (Sec. (4).

${ }_{83}$ We have implemented a prototype multimodal display sys${ }_{84}$ tem using normal and relief maps and performed both quali85 tative and quantitative evaluations of its effectiveness on per${ }_{86}$ ceptual quality of the VR experience and objective measures ${ }_{87}$ on task completion (Sec. 5). A user study suggests that normal ${ }_{88}$ maps can serve as an effective, unified texture representation for ${ }_{89}$ seamlessly integrated multi-sensory display and the resulting 90 system generally improves task completion rates with greater ${ }_{91}$ ease over use of a single modality alone. A second user study ${ }_{92}$ suggest that relief maps are also an effective representation of ${ }_{93}$ fine detail, with an improvement in sensory cohesiveness over ${ }_{94}$ normal maps.

\section{2. Previous Work}

Normal maps and relief maps are used throughout this pa${ }_{97}$ per as representations of fine detail of the surface of objects ${ }_{98}$ Normal maps were originally introduced for the purposes of ${ }_{99}$ bump mapping, where they would perturb lighting calculations 100 to make the details more visibly noticeable [2]. Relief mapping 101 uses both depths and normals for more complex shading [6, 7] 102 Numerous other texture mapping techniques exist as well. Dis${ }_{103}$ placement mapping, parallax mapping, and a number of more 104 recent techniques use height maps to simulate parallax and oc105 clusion [1, 4, 5]. A recent survey goes into more detail about 106 many of these techniques [11]. Mapping any of these textures 107 to progressive meshes can preserve texture-level detail as the 108 level-of-detail (LOD) of the mesh shifts [3].

109 Height maps mapped to object surfaces have been used to 110 modify the behavior of simple collisions in rigid-body simula111 tions [12]. We are not aware of similar work done using normal 112 maps aside from our own.

${ }_{113}$ In haptic rendering, a 3D object's geometries and textures 114 can be felt by applying forces based on point-contacts with the 115 object [13, 14]. Complex objects can also be simplified, with 116 finer detail placed in a displacement map and referenced to pro117 duce accurate force and torque feedback on a probing object [8]
${ }_{118}$ The mapping of both normal and displacement maps to simpli119 fied geometry for the purposes of haptic feedback has also been 120 explored [15]. Dynamic deformation textures, a variant of dis${ }_{121}$ placement maps, can be mapped to create detailed objects with 122 a rigid center layer and deformable outer layer. The technique ${ }_{123}$ has been extended to allow for 6-degree-of-freedom (DOF) hap124 tic interaction with these deformable objects [16]. A common 125 approach to force display of textures is to apply lateral force 126 depending on the gradient of a height map such that the user 127 of the haptic interface feels more resistance when moving "up128 hill" and less resistance when moving "downhill" [17, 18]. Our 129 approach to haptic rendering of textures applies force feedback 130 to simulate the presence of planes which reproduce this effect, 131 and similarly we use a simplified model for interaction with dy132 namic rigid-body objects.

${ }_{133}$ Modal analysis and synthesis are commonly used techniques 134 for synthesizing realistic sound [19]. Modal synthesis has been 135 integrated with rigid-body physics simulators in order to pro${ }_{136}$ duce contact sounds that synchronize with collision events. To 137 handle objects with arbitrary geometry, they can be decomposed 138 with finite element methods [20]. Further speed optimizations ${ }_{139}$ can be made based on psychoacoustics, such as mode compres140 sion and truncation [21]. We synthesize transient impact sounds ${ }_{141}$ by directly using this technique.

142 Sounds created by long-lasting contacts between objects re${ }_{143}$ quire some additional effort. Fractal noise is a common way 144 of representing the small impacts generated during rolling and 145 scraping [22]. We perform sound synthesis for lasting sounds 146 by using the framework for synthesizing contact sounds between 147 textured objects [9]. This work introduced a multi-level model ${ }_{148}$ for lasting contact sounds combining fractal noise with impulses ${ }_{149}$ collected from the normal maps on the surfaces of the objects 150 This application of normal maps to sound generation without ${ }_{151}$ similar application to rigid-body dynamics causes noticeable ${ }_{152}$ sensory conflict between the produced audio and visible physi${ }_{153}$ cal behavior.

\section{3. Overview and Texture Map Representation}

155 Our system uses three main components to create a virtual 156 scene where a user can experience through multiple modali157 ties of interaction. A rigid body physics simulator controls the 158 movement of objects. The only form of user input is through 159 a haptic device, which also provides force feedback to stimu${ }_{160}$ late the sense of touch. Finally, modal sound synthesis is used ${ }_{161}$ to dynamically generate the auditory component of the system. 162 In this section, we briefly cover the details of texture mapping, ${ }_{163}$ discuss haptic illusions and justify the use of texture represen164 tations, then describe each of these components using normal 165 maps as the representation of detail. The relief map representa${ }_{166}$ tion is covered in greater detail in Section 4 .

\section{3.1. Normal and Relief Maps}

${ }_{168}$ Normal maps are usually stored as RGB images, with the ${ }_{169}$ color values encoding vectors normal to the details of the sur170 face they are mapped to. Refer to Figure 1 for an example. It is 


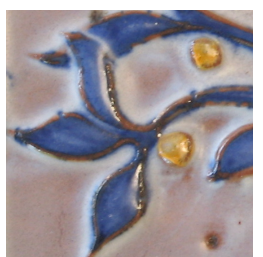

Color map

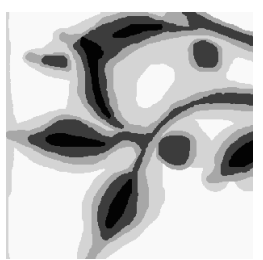

Depth map

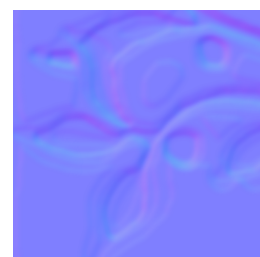

Normal map

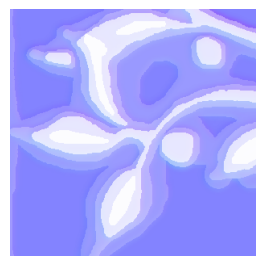

Relief map
Figure 1: Texture map example. RGB values encode normal vectors in each texel. In relief maps, the alpha value encodes depth information.

171 common practice to create normal maps directly corresponding 172 to a color map, such that the color map can be referenced at a lo173 cation to get a base color and the normal map can be referenced 174 at the same location for the corresponding normal vector.

175 Relief mapping is a technique for rendering textured sur176 faces using additional depth information. It is usually imple177 mented on GPUs and can be briefly described as computing 178 intersections with the height-field defined by the depth values 179 using rays from the camera to each pixel [7]. Ray casting lets 180 relief-mapped surfaces properly handle self-occlusion, and ex${ }_{181}$ tra ray casts from a light source enable self-shadowing. Since ${ }_{182}$ rays are cast from the camera, proper perspective is maintained 183 as the camera looks at the textured surface from different angles ${ }_{184}$ Our surfaces are rendered using relief mapping, so we refer to 185 their textures as "relief maps", though the same texture could 186 be used for parallax occlusion mapping or for displacements on ${ }_{187}$ GPU-tessellated surfaces.

188 Our relief maps contain their depth information in the al189 pha channel of the image. In the alpha channel, a value of 190 zero (black, entirely transparent) means the texel is at its high191 est, exactly along the geometry of the mapped object. Larger 192 values (tending towards white/visible) indicate that the texel is 193 recessed inside the object. Much like sculpted relief artwork 194 relief maps can only cut into the surface; they cannot raise a 195 texel outside the object's geometry. The maximum depth as a 196 percentage of mapped object dimensions can be set individually 197 for each relief map.

198 Depending on the resolution of the texture image and the 199 surface area of the object it is mapped to, a normal or relief 200 map can provide very fine detail about the object's surface. As 201 we describe in this paper, this detail — while still an approxima202 tion of a more complex surface-is sufficient to replicate other ${ }_{203}$ phenomena requiring knowledge of fine detail.

\section{3.2. Design Consideration}

205 Next we discuss various consideration in choosing texture 206 maps as our representation of fine detail, beginning with a dis207 cussion on haptic perception.

\section{3.2.1. Haptic Illusions}

209 Perceptual illusions, including visual, haptic and auditory, 210 have been explored in virtual reality for immersing users in 211 computer generated environments through multi-sensory dis212 play. For example, bump mapping can be regarded as a vi213 sual illusion where a user who is expecting to see depth in a 214 bump-mapped surface may interpret the shading as depth. Hap215 tic illusions can be roughly defined as when a haptic stimulus 216 is applied under specific conditions that change the perception 217 of that stimulus. A classic example is the size-weight illusion 218 in which a participant lifts two boxes of equal weight and un${ }_{219}$ equal sizes and perceives the smaller box to be heavier. There 220 are many types of haptic illusions, which have been well docu221 mented and catalogued [23].

222 There are some real-world examples of haptic illusions which 223 are relevant for simulating slope and depth. In the "curved ${ }_{224}$ plate" illusion, a flat edge rolled over a fingertip at about $1 \mathrm{~Hz}$ 225 produces the sensation that the edge is curved. As described ear226 lier, previous work on simulating haptic textures also relies on 227 haptic illusions: applying only lateral forces to a haptic probe ${ }_{228}$ can create the sensation of a vertical height difference.

${ }_{229}$ In these illusions, the changing direction of normal force 230 creates the illusion of curvature. That is, the normal vector is an 231 important haptic cue for curvature. Texture maps with normal 232 vectors provide exactly that information, and therefore should ${ }_{23}$ be able to simulate the curvature of a more complicated surface ${ }_{234}$ through haptic illusions. This observation forms the hypothesis 235 of our exploration of texture representations.

\section{3.2.2. Choice of Representation}

${ }_{237}$ On top of providing an important haptic cue, normal vectors 238 have additional advantages over alternative options. Using very 239 high-resolution geometry would automatically produce many 240 of the desired effects, but the performance requirements for in241 teractive 3D applications significantly reduces their viability in 242 our early deliberation. This is especially important to consider 243 in AR and VR applications, where real-time performance must 244 be maintained while possibly operating on a low-end mobile 245 phone or head mounted display.

${ }_{246}$ Other texture map information may also be considered, such 247 as height (or displacement) maps. For sound, Ren et al. [9] used 248 normal maps because the absolute height does not affect the re249 sulting sound; it is the change in normal which causes a single 250 impulse to produce meso-level sound. With regard to force dis251 play of textured surfaces, the Sandpaper system [18] has been 252 a popular and efficient method for applying tangential forces to 253 simulate slope based on a height map. Using normal vectors we 254 can instead scale a sampled normal vector to produce the same 255 normal and tangential forces. Rigid body collision response 256 also depends entirely on normal vectors.

${ }_{257}$ Since each component of the system depends directly on 258 the normals, a normal map representation emerges as the nat259 ural choice. An added convenience is that normal maps are 260 widely supported (including mobile games) and frequently in${ }_{261}$ cluded alongside color maps. Although normal maps contain 262 the most important cues for multimodal interaction, we would 
${ }_{263}$ like to evaluate how much benefit is gained from combining nor264 mals with depth information. Relief mapping uses both for vi265 sual rendering and has become more common alongside GPUs, 266 So relief maps provide a useful starting point for considering 267 depth in multimodal interaction with textures. The application 268 needs, the performance requirement, and the wide availability 269 and support on commodity systems all contribute to our adop270 tion of normal maps and relief maps as the mapping techniques 271 in this work.

\section{3.3. Rigid Body Dynamics}

273 In order to simulate the movement of objects in the virtual 274 scene, we use a rigid body dynamics simulator. These simula275 tors are designed to run in real time and produce movements of 276 rigid objects that visually appear believable.

277 Rigid body dynamics has two major steps: collision detec278 tion and collision response. Collision detection determines the 279 point of collision between two interpenetrating objects as well 280 as the directions in which to apply force to most quickly sepa${ }_{281}$ rate them. Modifying the normals of an object, as we do with 282 normal maps, does not affect whether or not a collision occurs. ${ }_{283}$ This is a significant limitation of a normal map representation 284 without any height or displacement information.

There are numerous algorithms for collision resolution, which ${ }^{328}$ ${ }_{286}$ determines how to update positions and/or velocities to sepa${ }_{287}$ rate the penetrating objects. In impulse-based approaches, col288 lisions are resolved by applying an impulse in the form of an in289 stantaneous change in each objects' velocity. Considering a sin290 gle object's velocity vector $\mathbf{v}, \Delta \mathbf{v}$ is chosen to be large enough 291 So that the objects separate in the subsequent timesteps. The 292 change in velocity on an object with mass $m$ is computed by 293 applying a force $f$ over a short time $\Delta t$ in the direction of the ${ }_{294}$ geometric normal $\mathbf{n}_{\mathbf{g}}$ of the other colliding object:

$$
\Delta \mathbf{v}=\frac{f \Delta t}{m} \mathbf{n}_{\mathbf{g}}
$$

295 This process is highly dependent on the normal vectors of each 296 object, and other collision resolution approaches have this same 297 dependency.

\section{3.3.1. Modifying Collision Behavior with Normal Maps}

299 We focus on simulating collisions between small dynamic 300 objects and large textured surfaces whose details would have a 301 large effect on the dynamic object. To get an intuitive under302 standing of the behavior we seek to replicate, imagine a marble ${ }_{303}$ rolling on a brick-and-mortar floor. When the marble rolls to 304 the edge of a brick, the expected behavior would be for it to fall 305 into the mortar between bricks and possibly end up stuck at the 306 bottom.

307 The level of detail needed to accurately recreate these dy308 namics with a conventional rigid body physics engine is too 309 fine to be interactively represented with a geometric mesh, es310 pecially with large scenes in real-time applications. A normal 311 map contains the appropriate level of detail and is able to repre312 sent the flat brick tops and rounded mortar indentations.

In order to change the behavior of collisions to respect fine ${ }_{314}$ detail, our solution is to modify the contact point and contact

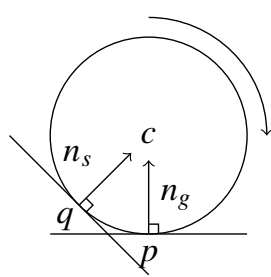

Figure 2: Contact point modification on a rolling ball: given the contact point $\mathbf{p}$ and sampled normal $\mathbf{n}_{\mathbf{s}}$, we want to simulate the collision at point $\mathbf{q}$.

315 normal reported by the collision detection step. This is an extra ${ }_{316}$ step in resolving collisions, and does not require any changes to 317 the collision detection or resolution algorithms themselves.

The contact normal usually comes from the geometry of the 319 colliding objects, but the normal map provides the same infor320 mation with higher resolution, so our new approach uses the 321 normal map's vectors instead. Given the collision point on the 322 flat surface, we can query the surface normal at that point and ${ }_{323}$ instruct the physics engine to use this perturbed normal instead 324 of the one it would receive from the geometry. One side effect 325 of using the single collision point to find the perturbed normal 326 is that it treats the object as an infinitely small probe.

\section{3.3.2. Rolling Objects and Collision Point Modification}

There is a significant issue with this technique when simu329 lating rolling objects. Refer to Figure 2 for an example. Two 3 so planes are shown, the horizontal one being the plane of the ${ }_{331}$ coarse geometry and the other being the plane simulated by the ${ }_{332}$ perturbed normal. Note that the contact points with the rolling ${ }_{3 з 3}$ ball differ when the plane changes. The vector $\mathbf{n}_{\mathbf{s}}$ shows the di334 rection of the force we would ideally like to apply. If we were 335 to apply that force at the original contact point $\mathbf{p}$, the angular 336 velocity of the sphere would change and the ball would begin ${ }_{337}$ to roll backwards. In practice, this often results in the sphere ${ }_{338}$ rolling in place when it comes across a more extreme surface 339 normal. Instead, we use the sphere radius $r$, the perturbed sur340 face normal $\mathbf{n}_{\mathbf{s}}$, and the sphere center $\mathbf{c}$ to produce the modified ${ }_{341}$ contact point $\mathbf{q}$ :

$$
\mathbf{q}=\mathbf{c}-(r \mathbf{n})
$$

342 This modification applies the force directly towards the center 343 of mass and causes no change in angular velocity, but is less 344 accurate for large spheres and extreme normal perturbations.

This contact point modification is important for perceptu${ }_{346}$ ally believable rolling effects. Shapes other than spheres do not 347 have the guarantee that the contact point will be in the direction ${ }_{348}$ of the $\mathbf{c}-\mathbf{n}$ vector, so this does not apply in the general case. ${ }_{349}$ Generally, we can simply modify the normal without changing 350 the contact point. In the case of relief maps, the true collision 351 points and contact normals can be determined, so this correc352 tion is unnecessary.

\section{3.4. Haptic Interface}

We have designed our system to use a PHANToM Desktop 355 haptic device [24]. This device can measure 6-DOF motion: 356 three translational and three rotational, but only display 3 -DOF 


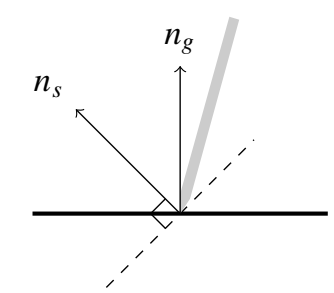

Figure 3: Haptic force is applied in the direction of the sampled normal $\mathbf{n}_{\mathrm{s}}$ instead of the geometric normal $\mathbf{n}_{\mathbf{g}}$.

357 forces (i.e. no torques). We have chosen to represent the PHAN${ }_{358}$ ToM as a pen inside the virtual environment, which matches 359 the scale and shape of the grip. While we could use forces de360 termined by the rigid-body physics engine to apply feedback, ${ }_{361}$ the physics update rate (about $60 \mathrm{~Hz}$ ) is much lower than the 362 required thousands of $\mathrm{Hz}$ needed to stably simulate a hard sur363 face.

${ }_{364}$ We simulate the textured surface by projecting the tip of the 365 PHANToM Desktop grip onto the surface in the direction of the 366 coarse geometry's normal. The fine surface normal is queried 367 and interpolated from nearby normal map vectors. The PHAN368 ToM simulates the presence of a plane with that normal and 369 the projected surface point. Given the normal vector sampled 370 from the normal map $\mathbf{n}_{\mathbf{s}}$ and pen tip position projected onto the 371 surface $\mathbf{p}$, the equation modeling this plane is:

$$
\left(\mathbf{n}_{\mathbf{s}} \cdot(x, y, z)\right)-\left(\mathbf{n}_{\mathbf{s}} \cdot \mathbf{p}\right)=0
$$

372 The PHANToM now needs to apply the proper feedback force ${ }_{373}$ to prevent the pen's tip from penetrating into the plane. This 374 is accomplished using a penalty force, simulating a damped 375 spring pulling the point back to the surface. Using the modi376 fied normal vector, the simulated plane serves as a local first 377 order approximation of the surface. Note that while the slopes 378 of the planes produced by the PHANToM can vary significantly 379 based on the normal map, at the position of the pen the plane ${ }_{380}$ will coincide with the surface. This is illustrated in Figure 3 , 381 where the simulated plane intersects the geometric plane at the 382 collision point. This creates an illusion of feeling a textured sur383 face while keeping the pen in contact with the flat underlying 384 surface geometry.

${ }_{385}$ With this technique, stability can be concern in some cases 386 Most noticeably, in steep and narrow V-shaped valleys, a user ${ }_{387}$ pushing down on the surface might cause the tip of the pen to 388 oscillate between the valley sides. Users sliding the pen rapidly 389 across bumpy surfaces may also feel forces that are stronger and 390 more abrupt than they would expect. We have mainly mitigated 391 these concerns by smoothing the normal maps and scaling down 392 the penalty forces. A side effect is that the surfaces end up 393 feeling slightly smoother and softer, though we have found this 394 an acceptable tradeoff for improved stability.

395 We use a simplified model to interact with dynamic objects 396 The PHANToM's corresponding pen appearance in the environ397 ment is added as an object in the rigid-body physics simulator. ${ }_{398}$ Whenever this pen comes in contact with a dynamic object, the 399 physics simulator computes the forces on the objects needed to 400 separate them. We can directly apply a scaled version of this 401 force to the haptic device. This ignores torque as our 3-DOF
402 PHANToM can only apply translational forces. This approach ${ }_{403}$ is fast, simple, and lets the user push and interact with objects 404 around the environment.

\section{3.5. Sound Synthesis}

406 Sound is created due to a pressure wave propagating through 407 a medium such as air or water. These waves are often produced 408 by the vibrations of objects when they are struck, and human 409 ears can convert these waves into electrical signals for the brain 410 to process and interpret as sound. One of the most popular ${ }_{411}$ physically-based approaches to modeling the creation of sound 412 is modal sound synthesis, which analyzes how objects vibrate ${ }_{413}$ when struck at different locations to synthesize contact sounds.

\section{${ }_{414}$ 3.5.1. Modal Analysis and Synthesis Background}

415 In order to perform modal analysis, we represent the objects 416 using a discretized representation such as a spring-mass system ${ }_{417}$ or a tetrahedral mesh. The dynamics of the object can be repre${ }_{418}$ sented with the system of differential equations:

$$
\mathbf{M} \ddot{\mathbf{r}}+\mathbf{C} \dot{\mathbf{r}}+\mathbf{K r}=\mathbf{f}
$$

${ }_{419} \mathbf{r}$ is a vector of displacements from the given starting positions, 420 which are assumed to be at rest. $\mathbf{f}$ is the vector of external forces ${ }_{421}$ applied to the system. $\mathbf{M}$ and $\mathbf{K}$ are the mass and stiffness ma422 trices, respectively, which describe the distribution of mass and ${ }_{423}$ connectivity of the object. For the damping matrix $\mathbf{C}$, we use ${ }_{424}$ Rayleigh damping which expresses $\mathbf{C}$ as a linear combination 425 of $\mathbf{M}$ and $\mathbf{K}$.

426 This system of equations can be decoupled to produce a 427 bank of modes of vibration. The equation for each mode is 428 a standard damped oscillator, which vibrates at a certain fre429 quency and decays exponentially over time. Almost all of the 430 complex calculations are dependent only of the properties of 431 the objects and therefore can be precomputed and stored.

432 Sound synthesis at runtime is done in two steps. When an 433 object is struck, the modes of vibration are excited depending ${ }_{434}$ on the strike's location and direction. Once the vibrations begin, 435 the modes are sampled and updated at around 44, $100 \mathrm{~Hz}$ to ${ }_{436}$ create perceptually realistic sound. For more details on modal ${ }_{437}$ analysis and synthesis, refer to the work of O'Brien et al. for 438 a FEM approach using tetrahedral meshes [20] or the work of ${ }_{439}$ Raghuvanshi and Lin for a spring-mass approach [21].

\section{3.5.2. Textures and Lasting Sounds}

441 Modal synthesis works well for generating sound that varies 442 for each object, material, and impulse. However, for long-lasting ${ }_{443}$ collisions such as scraping, sliding, and rolling, the sound pri${ }_{444}$ marily comes from the fine details of the surface which are not ${ }_{445}$ captured in the geometry of the input mesh when using texture 446 maps. We adopt the method by Ren et al. [9], which uses three 447 levels of detail to represent objects, with normal maps provid448 ing the intermediate level of detail.

At the macro level, the object is represented with the pro450 vided triangle mesh. The first frame in which a collision is 
451 detected, it is considered transient and impulses are applied ac452 cording to conventional modal synthesis. If the collision per${ }_{453}$ sists for multiple frames, we instead use the lower levels de454 scribed below.

455 Even surfaces that look completely flat produce rolling, slid456 ing, and scraping sounds during long-lasting collisions. The 457 micro level of detail contains the very fine details that produce 458 these sounds and are usually consistent throughout the material. 459 Sound at this level is modeled as fractal noise. Playback speed 460 is controlled by the relative velocity of the objects, and the am${ }_{461}$ plitude is proportional to the magnitude of the normal force.

462 The meso level of detail describes detail too small to be effi${ }_{463}$ ciently integrated into the triangle mesh, but large enough to be 464 distinguishable from fractal noise and possibly varying across 465 the surface. Normal maps contain this level of detail, namely 466 the variation in the surface normals. This sound is produced by 467 following the path of the collision point over time. Any time 468 the normal vector changes, the momentum of the rolling or slid469 ing object must change in order to follow the path of that new 470 normal. This change produces an impulse which can be used 471 alongside the others for modal synthesis. This can be mathe 472 matically formulated as follows.

473 Given an object with mass $m$ moving with tangent-space ve474 locity vector $\mathbf{v}_{\mathbf{t}}$ along a face of the coarse geometry with normal 475 vector $\mathbf{n}_{\mathbf{g}}$ whose nearest normal map texel provides a sampled 476 normal $\mathbf{n}_{\mathbf{s}}$, the component of the momentum orthogonal to the 477 face $\mathbf{p}_{\mathbf{n}}$ is:

$$
\mathbf{p}_{\mathbf{n}}=m\left(-\frac{\mathbf{v}_{\mathbf{t}} \cdot \mathbf{n}_{\mathbf{s}}}{\mathbf{n}_{\mathbf{g}} \cdot \mathbf{n}_{\mathbf{s}}}\right) \mathbf{n}_{\mathbf{g}}
$$

478 This momentum is calculated every time an object's contact 479 point slides or rolls to a new texel, and the difference is ap480 plied as an impulse to the object. More extreme normals or a 481 higher velocity will result in higher momentum and larger im482 pulses. Whenever objects are in collision for multiple frames, ${ }_{483}$ both the micro-level fractal noise and the meso-level normal 522 ${ }_{484}$ map impulses are applied, and the combined sound produces 485 the long-lasting rolling, sliding, or scraping sound.

\section{${ }_{486}$ 4. Relief Map Representation}

${ }_{487}$ As an extension to the modalities described above which 488 rely solely on the surface's normal vectors, we have also ex489 plored how a relief map's depth information can be incorpo490 rated to improve each component. In this section, we explain 491 these differences.

\section{4.1. Modifying Collision Behavior with Relief Maps}

493 When discussing rigid body physics with a normal map, we 494 mentioned that collision detection remained unchanged while 495 collision resolution required modification. With relief maps' ${ }_{496}$ depth information, collision detection now requires additional 497 steps, as now objects may penetrate inside the geometry of a 498 surface as long as they stay outside the recessed relief surface. 499 Again focusing on the situation where a small object collides

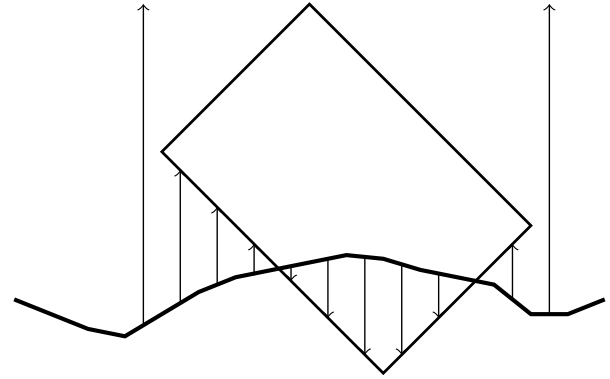

Figure 4: A rectangle colliding with a 1D relief map. Wherever arrows point downwards, the distance is negative and there is a collision.

500 with a large textured surface, the problem is collision detec501 tion between an object and a height map. We adopt a similar 502 approach described by Otaduy et al. for computing directional 503 penetration depth between two textured objects [8].

504 In general, the penetration depth between two colliding ob505 jects is the shortest distance one of the objects would have to 506 move in order to separate themselves. The directional penetra507 tion depth is the penetration depth where the objects can only 508 move along one specified axis. Computing the general penetra509 tion depth between finely-detailed objects can be prohibitively 510 slow for interactive applications. Directional penetration depth 511 can be used in place of general penetration depth, sacrificing 512 accuracy for speed, which is more appropriate for our goals.

${ }_{513}$ The GPU-based method proposed by Otaduy et al. for com514 puting directional penetration depth is to represent each collid515 ing object as a height map perpendicular to the specified direc516 tion. These height maps are aligned with one another so that the 517 distance between the objects at some point is the difference in 518 height between two matching height map texels. Wherever the 519 distance between objects is negative, there is a collision. The 520 most negative distance value can then be reported as the direc521 tional penetration depth.

522 In our case, the large plane textured with a relief map is al523 ready a height map perpendicular to the normal vector of the ${ }_{524}$ plane. In order to adopt a similar technique on any CPU (and ${ }_{525}$ GPU), we need to convert the colliding object into a height map 526 of its own. We primarily accomplish this by projecting the ${ }_{527}$ object onto the plane and rasterizing the result with the same 528 resolution as the relief map. The depth information from that ${ }_{529}$ process can then be used as the object's height map. The dif530 ference between the relief map's depth and the object's height ${ }_{531}$ map is the distance between them, and one or more collision 532 points can be found by searching for negative distances. The 533 collision points and the normal vectors sampled from the relief ${ }_{534}$ map at the same locations can then be passed to the collision ${ }_{535}$ resolution solver.

A simple example is illustrated in Figure 4, where a rect537 angular object is colliding with a 1D relief map. Each arrow 538 points from a relief map texel to the corresponding texel of the ${ }_{539}$ rasterized object height map, where upwards arrows are posi540 tive distance values and downwards arrows are negative. The 541 most negative distance values would be reported as collision 542 points. Since the points are found through a sampling process, 
${ }_{543}$ there is naturally a tradeoff between speed and accuracy: each 594 5.1. Performance Analysis

544 sample takes time to compute but contributes to finding a more 545 accurate collision point.

\section{4.2. Haptic Interface with Relief Maps}

595 The sound synthesis module generates samples at $44100 \mathrm{~Hz}$, 596 the physics engine updates at $60 \mathrm{~Hz}$, and while the PHANToM 597 hardware itself updates at around $1000 \mathrm{~Hz}$, the surface normal 598 is sampled to create a new plane once per frame. On a com547 For haptic interaction through the PHANToM, as with rigid 599 puter with an Intel Xeon E5620 processor and 24GB RAM, the 548 body physics, the change is in collision detection and not reso- 600 program consistently averages more than 100 frames per sec549 lution. The tip of the pen is projected down in the direction of 601 ond. This update rate is sufficient for real-time interaction, with 550 the surface normal, but collision is only reported if the pen's tip 602 multi-rate updates [8, 9].

551 is below the relief map depth value. If there is a collision, the 603 552 simulated plane is created in exactly the same way as described 553 in the normal map section. With depth information, the pen can 554 follow the actual contours of the surface.

\section{4.3. Sound Synthesis with Relief Maps}

${ }_{556}$ With normal maps, it is necessary to track the change in ${ }_{557}$ the sampled normal vector to estimate the impulses felt by a 558 rolling, sliding, or scraping object for the purposes of sound 559 synthesis. In the case of a relief map with depth information, 560 we can compute significantly more accurate collision informa${ }_{561}$ tion, and with that comes significantly more accurate impulse 562 information. With the relief map collision detection described ${ }_{563}$ previously, we can directly take the impulses reported by the ${ }_{564}$ physics engine and apply them to the bank of modes of vibra565 tion to synthesize sound.

566 Since the physics engine properly takes into account the nor${ }_{567} \mathrm{mal}$ and depth information from the relief map, the resulting 568 impulses already account for the texture detail. Adding in the 569 same fractal noise to account for surface variations too small to 570 be captured by either texture representation produces realistic 571 long-lasting contact sounds.

\section{${ }_{572}$ 5. Implementation and Results}

573 We have described each component of our multimodal sys574 tem using texture maps. We implemented this prototype system 575 in C++, using NVIDIA's PhysX as the rigid body physics sim576 ulator, OGRE3D as the rendering engine, VRPN to communi577 cate with the PHANToM [25], and STK for playing synthesized 578 sound [26].

579 In our previous work, we discretized our objects using spring580 mass systems to perform modal analysis for sound synthesis [10]. ${ }_{581}$ For this paper, we instead use a finite element method represen582 tation using tetrahedral meshes. The difference between the rep${ }_{583}$ resentations is primarily that the spring-mass model represents ${ }_{584}$ objects as hollow shells with a given shell thickness, while us585 ing tetrahedral meshes properly represents the full volume of 586 objects. With either representation, the equation in Section 3.5 .1 587 is used, but matrices are constructed differently. This provides ${ }_{588}$ an improvement in accuracy over spring-mass discretizations 589 and only negatively impacts the runtime during the precompu590 tation step. All scenarios we created contained at least one tex591 tured surface acting as the ground of the environment, and only 592 its normal map was used to modify collision response, haptic ${ }_{593}$ display, or sound rendering. 


\begin{tabular}{lcccccc}
\hline & Mesh Size & Offline Memory & Runtime Memory & Physics Time & Visual Time & Haptic Time \\
\hline Normal Map & $10 \mathrm{~KB}$ & $2.7 \mathrm{MB}$ & $270 \mathrm{~KB}$ & $175 \mu \mathrm{s}$ & $486 \mu \mathrm{s}$ & $60 \mu \mathrm{s}$ \\
Relief Map & $110 \mathrm{~KB}$ & $1 \mathrm{~GB}$ & $18 \mathrm{MB}$ & $2.2 \mathrm{~ms}$ & $900 \mu \mathrm{s}$ & $60 \mu \mathrm{s}$ \\
Coarse Mesh & $4.5 \mathrm{MB}$ & $288 \mathrm{~GB}^{*}$ & $450 \mathrm{MB}^{*}$ & $3.0 \mathrm{~ms}$ & $2.1 \mathrm{~ms}$ & $-^{* *}$ \\
Fine Mesh & $19 \mathrm{MB}$ & $4500 \mathrm{~GB}^{*}$ & $1700 \mathrm{MB}^{*}$ & $4.9 \mathrm{~ms}$ & $7.0 \mathrm{~ms}$ & $-^{* *}$ \\
\hline
\end{tabular}

Table 1: Memory and timing results for our (texture-based) methods compared to a similarly detailed coarse mesh (66,500 vertices) and fine mesh (264,200 vertices). Entries marked with * are extrapolated values, since the memory requirements are too high to run on modern machines. Haptic time $(* *)$ was not measurable for triangle meshes due to an API limitation. Normal maps are able to achieve up to 25 times of runtime speedup and up to 6 orders of magnitude in memory saving.

\section{5.2. Normal Map Texture Identification User Study}

649 In order to evaluate the effectiveness of this multimodal sys650 tem, we conducted a user study consisting of a series of tasks ${ }_{651}$ followed by a questionnaire. One objective of this user study 652 was to determine the overall effectiveness of our system. For ${ }_{653}$ this study, only the normal map representation was used. A ${ }_{654}$ subject is interacting with the normal map through sight, touch, 655 and sound. If each of these components are well designed and 656 implemented, a subject should be able to identify the material 657 by multimodal interaction. The other goal is to see how well the 658 use of multiple senses helps to create a cohesive recognition of 659 the material being probed. Even if subjects find the haptic dis${ }_{660}$ play alone is enough to understand the texture of the material ${ }_{661}$ being probed, does adding sound cues speed up their process of 662 identifying textures or instead cause sensory conflict?

\section{5.2.1. Set-up}

664 Twelve participants volunteered to take part in this study 665 experiment. Each subject was trained on how to use the PHAN${ }_{666}$ ToM and was given some time to get used to the system by ${ }_{667}$ playing in a test scene (see Figure 7 top row). The subject then ${ }_{668}$ completed a series of six trials. In each trial, a material for the 669 surface was chosen at random, and all aspects of it except its 670 visual appearance were applied. That is, the subject would be 671 able to feel the surface's texture with the PHANToM, hear the 672 sound generated from ball and PHANToM pen contacts, and 673 see the rolling ball respond to ridges and valleys on the surface. ${ }_{674}$ The subject was able to cycle through each material's visual ap675 pearance (in the form of a texture) by pressing the button on 676 the PHANToM's grip. Their task was to select the material's 677 unknown visual appearance based on the multimodal cues re678 ceived.

679 The first three trials provided all three cues—sound, ball, 680 and pen - but in each of the remaining three trials only two of ${ }_{681}$ the three cues would be available. The subject would be in682 formed before the trial began if any cues were missing. The 683 subjects were recommended to use all available cues to make 684 their decision, but were otherwise unguided as to how to dis685 tinguish the materials. After the trials were completed, a short 686 questionnaire was provided for subjective evaluation and feed687 back.

${ }_{688}$ This study utilizes sensory conflict to guide the subjects to 689 correctly identify the visual appearance. If the multimodal cues ${ }_{690}$ present the sounds, haptic texture, and physical response of a ${ }_{691}$ metal surface with regular grooves, but the subject has currently

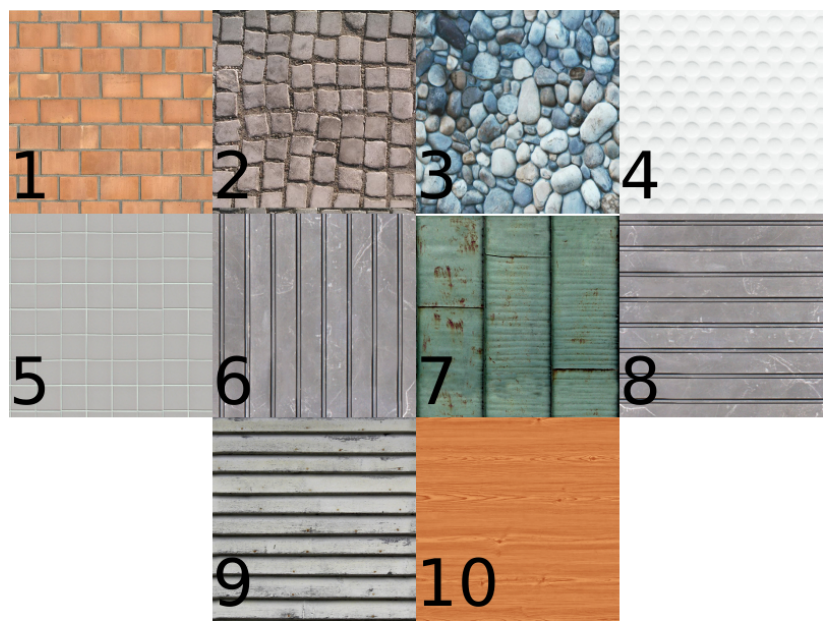

Figure 5: The available materials for the texture identification user study. 1-3 sounded like bricks, 4-5 sounded like porcelain, 6-8 sounded like metal, and 9-10 sounded like wood.

\begin{tabular}{lccc}
\hline & ID rate & Time $(\mathbf{s})$ & Ease (1-10) \\
\hline All modes & $78 \%$ & $38 \pm 18$ & $7.9 \pm 1.3$ \\
No sound & $81 \%$ & $46 \pm 45$ & $4.9 \pm 2.2$ \\
No haptics & $54 \%$ & $41 \pm 23$ & $3.6 \pm 1.8$ \\
No physics & $72 \%$ & $47 \pm 58$ & $6.4 \pm 2.6$ \\
\hline
\end{tabular}

Table 2: Results comparing effectiveness when limiting the available modes of interaction in the texture identification user study. "Ease" is evaluated by the subjects where 1 is difficult and 10 is easy. When using all modes of interaction, subjects were generally able to identify the material more frequently than when only using two modes and reported that they found identification to be easiest when all modalities of interaction were engaged.

692 selected the visual appearance of a flat, smooth wooden sur693 face, they should recognize the sensory conflict and reject the 694 wooden surface as the answer. Once the subject has selected 695 the correct visual appearance (grooved metal in this example), 696 they should feel relatively little sensory conflict and from that 697 realize they have found the answer.

Figure 5 shows the materials chosen for the user study. The 699 subjects were allowed to look at each of these textures before 700 the trials began, but were not able to feel or hear them. Some of 701 these were specifically chosen to be challenging to distinguish.

\section{5.2.2. Experimental Results}

703 In Table 2] we compare the results when varying which 704 modes of interaction are available to subjects. The ID rate is 705 the percentage of trials in which the subject was able to cor706 rectly identify the material, and the mean time only takes into 


\begin{tabular}{r|cccccccccc}
\hline & \multicolumn{10}{|c}{ Guesses (\%) } \\
ID & $\mathbf{1}$ & $\mathbf{2}$ & $\mathbf{3}$ & $\mathbf{4}$ & $\mathbf{5}$ & $\mathbf{6}$ & $\mathbf{7}$ & $\mathbf{8}$ & $\mathbf{9}$ & $\mathbf{1 0}$ \\
\hline $\mathbf{1}$ & $\mathbf{5 0}$ & 0 & 33 & 0 & 0 & 17 & 0 & 0 & 10 & 0 \\
$\mathbf{2}$ & 0 & $\mathbf{8 0}$ & 0 & 20 & 0 & 0 & 0 & 0 & 0 & 0 \\
$\mathbf{3}$ & 0 & 0 & $\mathbf{1 0 0}$ & 0 & 0 & 0 & 0 & 0 & 0 & 0 \\
$\mathbf{4}$ & 0 & 0 & 0 & $\mathbf{8 3}$ & 17 & 0 & 0 & 0 & 0 & 0 \\
$\mathbf{5}$ & 0 & 13 & 25 & 0 & $\mathbf{5 0}$ & 0 & 12 & 0 & 0 & 0 \\
$\mathbf{6}$ & 0 & 0 & 17 & 0 & 0 & $\mathbf{8 3}$ & 0 & 0 & 0 & 0 \\
$\mathbf{7}$ & 8 & 0 & 8 & 0 & 0 & 8 & $\mathbf{6 0}$ & 8 & 8 & 0 \\
$\mathbf{8}$ & 0 & 0 & 0 & 0 & 0 & 0 & 0 & $\mathbf{7 5}$ & 25 & 0 \\
$\mathbf{9}$ & 0 & 0 & 17 & 0 & 0 & 0 & 0 & 16 & $\mathbf{6 7}$ & 0 \\
$\mathbf{1 0}$ & 0 & 0 & 0 & 0 & 0 & 0 & 0 & 0 & 12 & $\mathbf{8 8}$ \\
\hline
\end{tabular}

Table 4: Confusion matrix showing the guesses made by subjects in the texture identification study. For all materials, a significant majority of subjects were able to identify the right materials.

707 account time for correct guesses. The "ease" was provided by 708 the subjects on the questionnaire, where they were asked to rate 709 on a scale from 1-10 how easy they found it was to identify the 710 material for each combination of modes of interaction. Higher ${ }_{711}$ "ease" scores mean the subject found it easier to identify the 712 material.

${ }_{713}$ In all cases, the identification rate was higher than $50 \%$, 714 and usually much higher than that. The loss of haptic feed715 back caused the largest drop in ID rate and ease. The loss 716 of sound actually improved material identification-although 717 the difference is not statistically significant-but subjects still 718 found identification to be much more perceptually challenging. 719 Two more noteworthy results were gathered from a subjec720 tive questionnaire, with results shown in Table 3 . Subjects were 721 asked how frequently they used each of the modes in identify722 ing the material. The subjects were also asked how well each 723 mode of interaction represented how they would expect the ma724 terials to sound or feel. These results could help explain the 725 low identification rate when haptics are disabled: most subjects 726 both relied heavily on tactile senses and found it be the most 727 accurate mode. The subjects considered the sound and physics 728 somewhat less accurate but still occasionally useful for deter729 mining the materials.

730 More detailed results from the study are presented in Ta${ }_{731}$ ble 4. An entry in row $i$ and column $j$ is the percentage of times 732 the subject was presented material $i$ and guessed that it was ma733 terial $j$. The higher percentages along the diagonal demonstrate 734 the high correct identification rate. Also note that in most cate735 gories there is no close second-place guess. The largest excep${ }_{736}$ tion is that $33 \%$ of the time material 1 (brick grid) was mistak ${ }_{737}$ enly identified as material 3 (pebbles), likely due to similarity 738 in both material sounds and patterns.

\section{7з9 5.2.3. Analysis}

740 Our analysis is largely based on comparing the results from ${ }_{741}$ interactions with different sets of modalities using a $t$-test to an742 alyze the difference between the modalities. In addition to the ${ }_{743} p$ value for statistical significance, we also use Cohen's effect 744 size $d$, defined as the difference between the means of two sam745 ples divided by their pooled standard deviation [27]. Effect size
746 is an important factor to consider alongside statistical signifi747 cance, explaining not just if there is a difference, but explaining 748 (in units of standard deviations) how large that difference actu749 ally is.

750 Due to the relatively low sample size in the study of each 751 material, many of the possible direct comparisons would not be 752 statistically significant. Therefore, for this study the reported ${ }_{753}$ statistics are based on combined data from all study materials; 754 we do not compare the result on each material to one another.

755 Between identification rates, there was no statistically sig756 nificant change when removing a mode $(p>.05)$, but the re757 moval of haptics came close with $p=.066$. The subjective 758 subject-reported values of ease and accuracy were generally 759 more significant. Subjects reported that they found material 760 identification to be more difficult when either sound or hap761 tics were removed in comparison to having all modes available $762(p<.05)$, but did not find identification more difficult when the ${ }_{763}$ physics modification was removed $(p>.05)$. Cohen's effect 764 size values $(d)$ of 1.66 for the removal of sound and 2.79 for the 765 removal of haptics suggest a very large change in perceptual 766 difficulty when removing these modes. Subjects also reported 767 that they found the haptics to be more accurate than physics or 768 sound $(p<.05)$, but did not find a significant difference in accu769 racy between physics and sound $(p>.05)$. Cohen's effect size 770 values of 1.02 comparing haptics to physics and 1.36 compar771 ing haptics to sound suggest a large difference in the perception 772 of how accurate these modes are.

773 Overall, these results demonstrate that each mode of inter774 action is effectively enabled through use of normal maps. Com775 bining multiple modes increases accuracy, which suggests that 776 the subjects are receiving cohesive, non-conflicting information 777 across their senses. This was a deliberately challenging study, 778 using materials which sounded similar and had similar geomet779 ric features and patterns. Furthermore, the task asked subjects 780 to carefully consider properties of materials not often noticed. ${ }_{781}$ Not many people take the time to consider the difference in fre782 quency distributions between the sounds of porcelain and metal, 783 but that distinction could have been important for these tasks. ${ }_{784}$ Within such a context, a 78\% rate for identifying the correct 785 material out of ten options appears rather promising, and signif786 icantly better than random selection.

\section{5.3. Normal and Relief Comparison User Study}

We now move on to discuss a second, separate user study. 789 In order to evaluate the effectiveness of the relief map represen790 tation, we conducted another user study where subjects com791 pared normal mapped surfaces to relief mapped surfaces. Since 792 the previous study found most of the benefit in the subjects' per793 ception of the surface, this study was largely designed to test the ${ }_{794}$ perceptual aspects of these representations.

\section{5.3.1. Set-up}

Twenty-two subjects volunteered to participate in this study, 797 primarily students with computer literacy in the age between 20 798 to 30 . The subjects were allowed to interact with six textured 799 surfaces, where, for each subject, three textures were randomly 


\begin{tabular}{lccccc|c}
\hline & Always & Frequently & Occasionally & Rarely & Never & Reported accuracy (1-10) \\
\hline Haptics & $88 \%$ & $0 \%$ & $6 \%$ & $0 \%$ & $6 \%$ & $9.3 \pm 0.9$ \\
Sound & $34 \%$ & $22 \%$ & $22 \%$ & $11 \%$ & $11 \%$ & $7.6 \pm 1.4$ \\
Physics & $29 \%$ & $6 \%$ & $47 \%$ & $6 \%$ & $12 \%$ & $7.3 \pm 2.6$ \\
\hline
\end{tabular}

Table 3: Texture identification study: Results from question asking how often subjects used each mode of interaction and question asking how well each mode represented the materials (10 is very accurate).

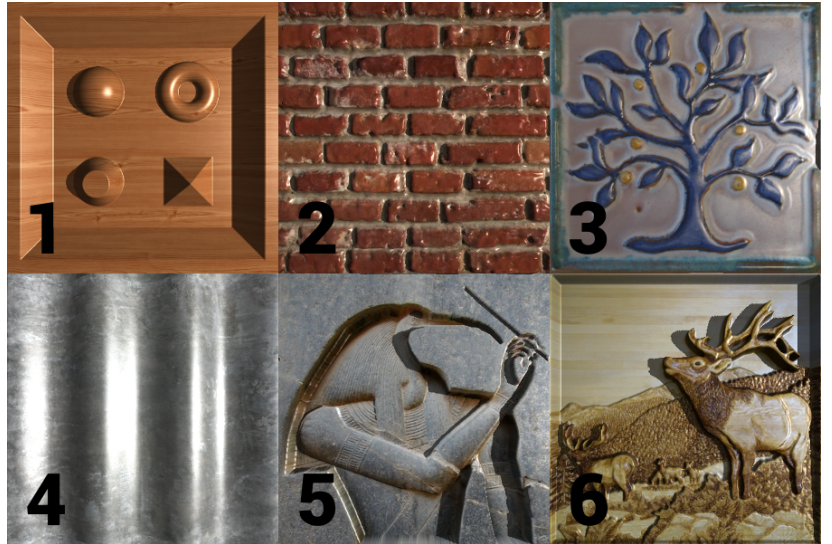

Figure 6: The available materials for the normal and relief map comparison user study. Material 2 and 5 sounded like stone; 3 sounded like ceramic tile; 4 sounded like metal; 1 and 6 sounded like wood.

800 selected to use the normal map representation and the remain801 ing three used the relief map representation. Much like in the ${ }_{802}$ previous user study, subjects controlled the PHANToM, which ${ }_{803}$ corresponded to a virtual pen that could strike the surface or a 804 rolling ball. Through this interaction the subjects would feel the 805 surface, watch the ball roll across the surface, and hear sound ${ }_{806}$ synthesized from the surface. Subjects were given as much time 807 as needed to interact with the textured surfaces, and were able to 808 switch between textures at will. Feedback was obtained through 809 a questionnaire in which subjects evaluated each texture, rating 810 the perceived realism of the visual appearance, how well each 811 mode of interaction matched what they would expect from the 812 visual appearance, and the overall quality of interaction.

813 Figure 6 shows the relief map versions of each surface cho814 sen for the user study. These were selected to provide a range 815 of complexity, depth, and materials. The subjects were allowed ${ }_{816}$ to spend as much time as needed to properly evaluate each sur817 face.

818 The subjects were not informed that some surfaces would 819 have relief maps and some would have normal maps, nor were 820 they specifically told to consider the depth of the surface. Fur${ }_{821}$ thermore, no subject ever saw both the normal and relief ver822 sions of the same surface, always one or the other. With the ${ }_{823}$ subjects largely going into the study unaware of the multiple 824 representations, we pose the following questions:
830 it more highly than the subjects interacting with its nor${ }_{831} \quad$ mal map equivalent?

${ }_{832}$ - How much, if any, does depth information help with re833 duction of sensory conflict?

\section{5.3.2. Experimental Results}

${ }_{835}$ A general way to look at the results is to, for each question, ${ }_{836}$ compare all responses (across all surface materials) to use of 837 normal maps vs. use of relief maps. This way can provide a gen${ }_{838}$ eral idea of which texture representation was preferred for each 839 mode of interaction. When subjects were asked how realistic 840 the surfaces appeared, how much the ball physics matched their 841 expectations, and how much the synthesized sound matched 842 their expectations, there was no significant difference between 843 normal maps and relief maps $(p>>.05)$. Cohen's effect size ${ }_{844}$ for each of these was no greater than 0.11 , further indicating 845 little distinction between the texture representations.

846 When subjects were asked how well the haptics matched 847 their expectations, there was weak evidence showing that sub848 jects preferred the relief maps $(p \approx .053)$, and Cohen's effect 849 size of .34 indicates some moderate preference of relief maps. 850 However, when subjects reported their overall perceived qual851 ity of interaction, they significantly favored relief maps over 852 normal maps $(p<.05)$, with Cohen's effect size of .36 further 853 suggesting a moderate preference of relief maps.

In Table 5, we show the results from comparing the two ver855 sions of each texture to one another. For each of the six surfaces, 856 the ratings from the subjects who were given the normal map 857 version are compared to the ratings from the subjects who were ${ }_{858}$ given the relief map version, and the table presents the $p$ values 859 and effect sizes for each category the subjects were questioned 860 about. See the beginning of Section 5.2.3 for a brief description ${ }_{861}$ of effect size. Notice that the results vary largely from surface 862 to surface.

Recall that, out of the six surfaces each subject experienced, 864 three at random were chosen to be normal maps and the other 865 three were relief maps. Comparing each subject's average nor866 mal map rating to that same subject's average relief map rating, ${ }_{867}$ we found that each subject tended to prefer their three relief 868 maps over their three normal maps $(p<.05)$.

\section{5.3.3. Analysis}

We can now revisit our originally posed questions, which 871 each involve different means of analyzing the data:

872 Accuracy and realism of relief maps. In order to assess the 873 overall quality of interaction with relief maps, we can consider 


\begin{tabular}{lccccccc}
\hline & & \multicolumn{7}{c}{ Surface } \\
& & $\mathbf{1}$ & $\mathbf{2}$ & $\mathbf{3}$ & $\mathbf{4}$ & $\mathbf{5}$ & $\mathbf{6}$ \\
\hline \multirow{2}{*}{ Visuals } & $p$ & .03 & .61 & .96 & .14 & .21 & .66 \\
& $d$ & .84 & -.21 & .03 & .65 & -.57 & .18 \\
\hline \multirow{2}{*}{ Physics } & $p$ & .80 & .64 & .38 & .83 & .08 & .56 \\
& $d$ & -.1 & .20 & -.4 & .09 & -.78 & .25 \\
\hline \multirow{2}{*}{ Sound } & $p$ & .31 & .84 & .47 & .27 & .07 & .14 \\
& $d$ & -.45 & -.09 & -.34 & .49 & -.83 & .65 \\
\hline \multirow{2}{*}{ Haptics } & $p$ & .03 & .70 & .77 & .03 & .002 & .002 \\
& $d$ & .9 & .16 & .16 & 1.03 & -1.42 & 1.44 \\
\hline \multirow{2}{*}{ Overall } & $p$ & .2 & .68 & .92 & .08 & .14 & .02 \\
& $d$ & .52 & .18 & .05 & .80 & -.65 & 1.02 \\
\hline
\end{tabular}

Table 5: For each of the six surfaces, subjects interacted with either the normal or relief map version of that surface's texture. This table contains results of $t$-tests for each surface and each modality determining whether there are significant differences between the subjects' responses for each texture representation. A small $p$ indicates a statistically significant difference. A positive $d$ value indicates that subjects prefer the relief map version; negative indicates a preference for the normal map.

874 the data in aggregate, regardless of surface or user. Based on 875 the subjects' ratings of the surfaces' overall quality across all 876 surfaces, on average subjects preferred relief maps over normal 877 maps. We also know that, despite not being informed of the 878 multiple representations, subjects significantly preferred their ${ }_{879}$ three randomly selected relief maps over their three normal 880 maps. This neglects the subjects' opinions on individual modes ${ }_{881}$ of interaction, but that will be discussed later in the context of 882 sensory conflict. When considered as a whole, relief maps were 883 considered to be of somewhat better overall quality.

${ }_{884}$ Comparisons between normal and relief map versions of 885 the same surface. In order to see how subjects compared dif886 ferent versions of the same surface, we now focus on the data 887 in Table 5, which groups ratings by surface. When broken up 888 in this way, we now see that results varied greatly from sur889 face to surface. For most surfaces and most modes of inter890 action, the differences in ratings were not statistically signifi${ }_{891}$ cant, and the effect sizes ranged from medium preference of the 892 normal map to medium preference of the relief map. Certain ${ }_{893}$ textures therefore may be more suitable for representation as re894 lief maps than others. For example, subjects often commented 895 that haptics and ball physics were unrealistic near vertical edges ${ }_{896}$ in a relief map (likely due to limitations of directional pene897 tration depth). Surface five contained many prominent near898 vertical edges, and subjects strongly preferred the normal map 899 version. Even though there is an average preference for relief 900 maps across all surfaces, this and other situational reasons for ${ }_{901}$ preferring a particular representation mean that the choice of 902 representation may need to be considered on a case-by-case ba903 sis.

${ }_{904}$ Reduction of sensory conflict. In order to assess sensory con905 flict, we now see if the results indicate that the experience as a 906 whole was more appealing than each separate modality would
907 indicate. Preferences were mixed when subjects were told to 908 rate a specific mode of interaction, but they rated the overall 909 quality of relief maps to be significantly higher than normal 910 maps. This suggests that when interacting with multiple modes 911 of interaction simultaneously, relief maps appear to produce 912 more cohesive multimodal interaction than normal maps. Nor${ }_{913}$ mal vectors already provided most of the cues for depth and 914 curvature, so adding depth information in the form of a relief 915 map only had a small effect on any one mode of interaction. It 916 is only when all modes are considered together that the com917 bined effect is significantly larger. While the overall quality of 918 interaction with reliefs maps may only be moderately better on 919 average and dependent on traits of the surface itself, this reduc920 tion in sensory conflict provides its own, possibly subconscious, ${ }_{921}$ advantages.

\section{5.4. Discussion}

\section{${ }_{923}$ 5.4.1. Applications}

${ }_{924}$ We demonstrate several possibilities on the potential use of ${ }_{925}$ normal and relief maps as unified representations for accelerat926 ing multimodal interaction in the supplementary video. Given ${ }_{927}$ the prevalence of texture mapping in numerous interactive 3D ${ }_{928}$ graphics applications (e.g. games and virtual environment sys929 tems), our techniques enable the users to interact with textured 930 objects that have extremely simple underlying geometry (such ${ }_{931}$ as flat surfaces) so that they would be able to observe consistent ${ }_{932}$ dynamic behaviors of moving textured objects, hear the result933 ing sounds from collisions between them, and feel the object ${ }_{934}$ contacts, as shown in Figure 7 (top row). The example of the 935 simplified pinball game in Figure 7(bottom right), balls rolling 936 down Lombard Street in San Francisco City in Figure 8, and let${ }_{937}$ ter blocks sliding down sloped surfaces with noise or obstacles 938 in Figure 7 are a few additional examples, where texture maps 939 can be incorporated into physics simulation with multimodal 940 display to provide a more cohesive, immersive experience with${ }_{941}$ out sensory disparity. Please see the supplementary video for 942 demonstration of these results.

\section{${ }_{943}$ 5.4.2. Comparison with Level-of-Detail Representations}

${ }_{944}$ While we have shown comparisons between normal maps 945 and high-resolution meshes as representations of fine detail, us946 ing multiple levels-of-detail when appropriate can also improve ${ }_{947}$ runtime performance [28, 29, 30]. These LOD meshes can also 948 reduce the complexity of the geometry while trying to retain 949 the most important features, as determined by perceptual met950 rics. Since human perception is limited, there may be no signif${ }_{951}$ icant perceptual benefit in using meshes past a certain quality, ${ }_{952}$ in which case the simplified version could be used throughout 953 for significant performance gain.

${ }_{954}$ However, there would be a number of challenges to over955 come in designing a multimodal LOD system. The metrics ${ }_{956}$ defining important visual features are known to be different than 957 the metrics defining important haptic features [31]. It remains 958 an open problem to create metrics for selecting important au959 dio features for switching between LODs in a multimodal sys960 tem. Furthermore, the haptic LOD meshes are different from 

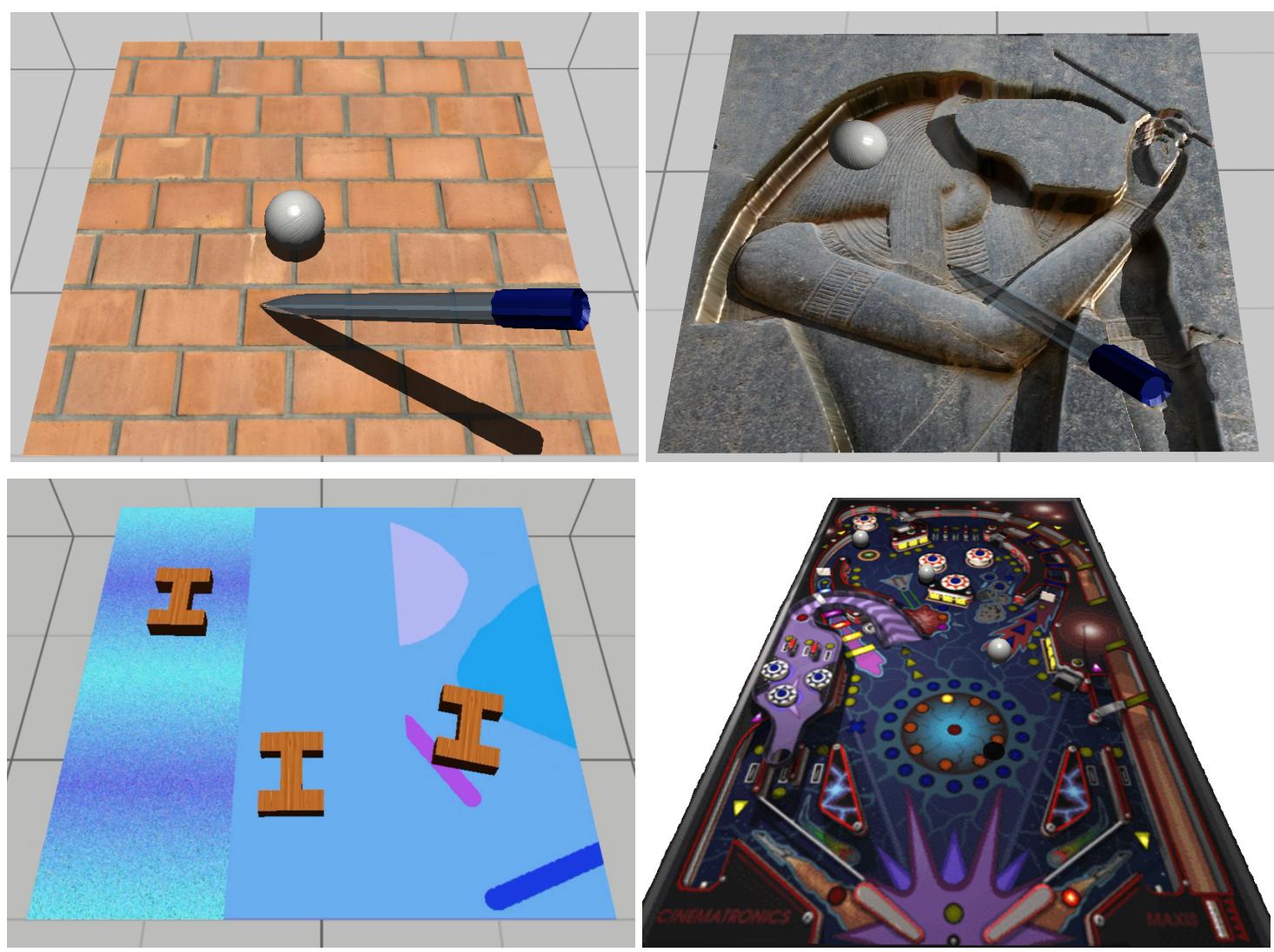

Figure 7: A selection of applications based on our system: a virtual environment with multimodal interaction with a normal map used in the texture identification user study (top left), multimodal interaction with a relief map used in the normal and relief map comparison user study (top right), letter blocks sliding down a normal-mapped surface (bottom left), and a pinball simulation on a normal-mapped flat plane (bottom right).

${ }_{961}$ LOD meshes for visual rendering [31], leading to significantly 962 higher memory requirements than texture-based representation 963 in general.

\section{6. Conclusion}

${ }_{965}$ In this paper, we presented an integrated system for multi966 modal interaction with textured surfaces. We demonstrated that 967 normal maps and relief maps can be used as unified representa968 tions of fine surface detail for visual simulation of rigid body 969 dynamics, haptic display and sound rendering. We showed 970 that in a system which uses normal maps to present fine de971 tail to subjects through multiple modes of interaction, subjects 972 are able to combine this information to create a more cohesive 973 mental model of the material they are interacting with. Our first 974 user evaluation result further provides validation that our sys975 tem succeeded in reducing sensory conflict in virtual environ976 ments when using texture maps. Our second user evaluation re977 sult demonstrates that relief maps, when chosen carefully, may ${ }_{978}$ produce a further reduction in sensory conflict.

979 We have now explored two different texture representations ${ }_{980}$ of fine detail, but some limitations should be addressed. Our ${ }^{1005}$ ${ }_{981}$ current implementation and studies limited the texture-mapped ${ }_{982}$ surfaces to single flat planes and we assume our multimodal ${ }_{983}$ method would translate gracefully to more complex shapes, as ${ }_{1000}^{1000}$ 984 techniques exist for visually rendering relief maps on arbitrary 1010 ${ }_{985}$ polygonal surfaces [7]. We have also only been detecting col- ${ }^{1011}$
986 lisions between static relief-mapped surfaces and dynamic non${ }_{987}$ relief-mapped objects. A more generalized and versatile system ${ }_{988}$ could consider the texture of both colliding textured objects, 989 even if both are dynamic, although performance may become 990 more of a limitation. Vectorial textures may be used to help 991 reducing the aliasing artifacts of relief maps in better render992 ing sharp edges. Additionally, our choice of haptic device has 993 limited our results to 3-DOF force feedback, though it should 994 be possible to compute torques with a slight extension of our 995 method.

996 For future research, it may be possible to explore the inte997 gration of material perception [32, 33] for multimodal displays 998 based on some of the principles described in this paper. Future 999 work may also attempt to generalize this system by addressing 1000 the limitations described. We hope this work will lead to further 1001 interest in development of techniques on minimizing sensory 1002 conflicts when using texture representations for interactive 3D ${ }_{1003}$ graphics applications, like AR and VR systems.

\section{Acknowledgments}

This research is supported in part by National Science Foun${ }_{1006}$ dation and the UNC Arts and Sciences Foundation.

[1] Cook RL. Shade trees. In Proceedings of the 11th Annual Conference on Computer Graphics and Interactive Techniques. SIGGRAPH '84; New York, NY, USA: ACM. ISBN 0-89791-138-5; 1984, p. 223-31. URL: http://doi.acm.org/10.1145/800031.808602 doi $10.1145 / 800031.808602$ 

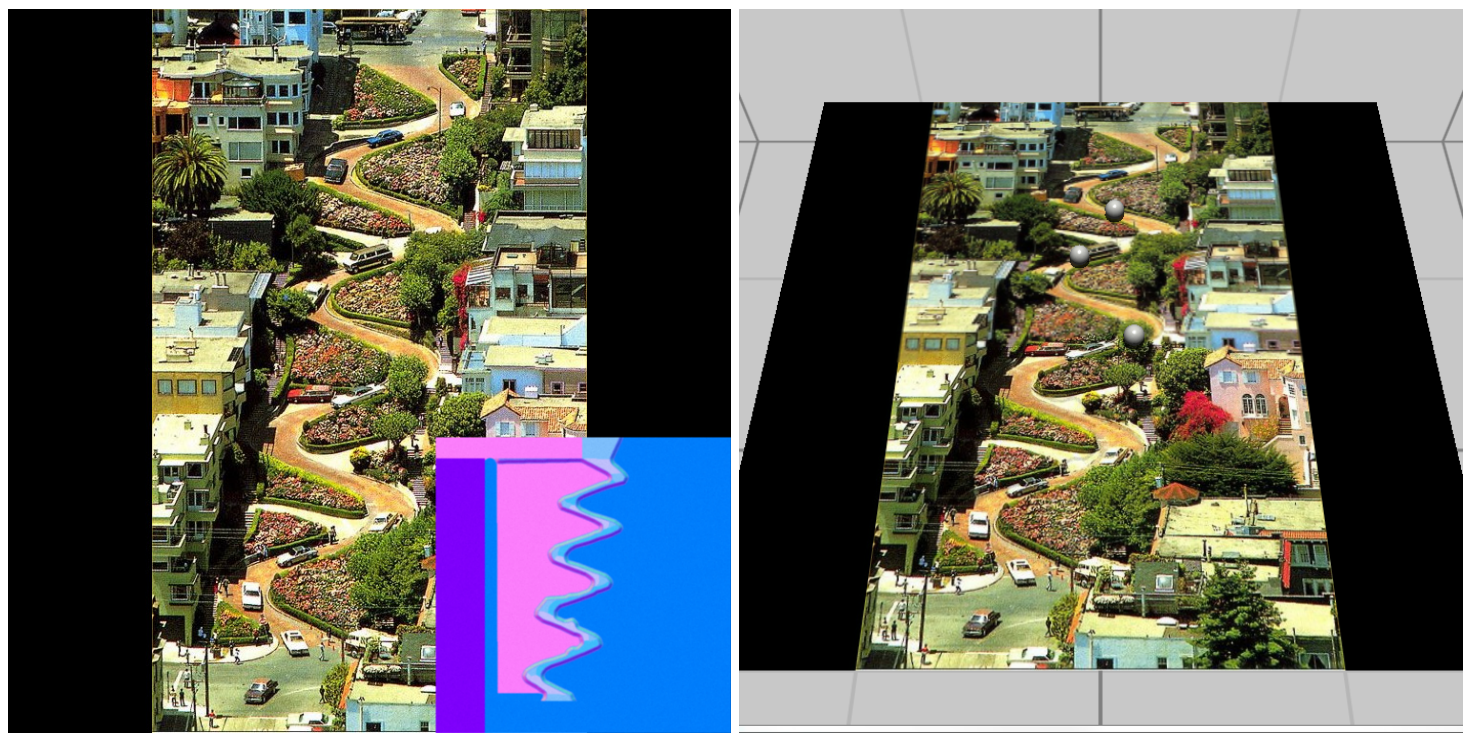

Figure 8: Lombard street color map with normal map (left) and mapped to a plane with rolling balls (right).

[2] Blinn JF. Simulation of wrinkled surfaces. GRAPH Comput Graph 1978;12(3):286-92. http://doi.acm.org/10.1145/965139.507101 doi $10.1145 / 965139.507101$ plification. In Proceedings of the 25th Annual Conference on 1063 Computer Graphics and Interactive Techniques. SIGGRAPH '98; 1064 New York, NY, USA: ACM. ISBN 0-89791-999-8; 1998, p. 1065 115-22. URL: http://doi.acm.org/10.1145/280814.280832 1066 doi $10.1145 / 280814.280832$

[4] Kaneko T, Takahei T, Inami M, Kawakami N, Yanagida Y, Maeda T, et al. 1068 Detailed shape representation with parallax mapping. In In Proceedings 1069 of the ICAT. 2001, p. 205-8.

[5] Tevs A, Ihrke I, Seidel HP. Maximum mipmaps for fast, accurate, 1071 and scalable dynamic height field rendering. In Proceedings of the 1072 2008 Symposium on Interactive 3D Graphics and Games. I3D '08; 1073 New York, NY, USA: ACM. ISBN 978-1-59593-983-8; 2008, p. 1074 183-90. URL: http://doi.acm.org/10.1145/1342250.1342279 1075 doi $10.1145 / 1342250.1342279$

6] Oliveira MM, Bishop G, McAllister D. Relief texture mapping. In 1077 Proceedings of the 27th Annual Conference on Computer Graphics 1078 and Interactive Techniques. SIGGRAPH '00; New York, NY, USA: 1079 ACM Press/Addison-Wesley Publishing Co. ISBN 1-58113-208-5; 2000, 1080 p. 359-68. URL: http://dx.doi.org/10.1145/344779.344947 1081 doi $10.1145 / 344779.344947$

[7] Policarpo F, Oliveira MM, Comba JaLD. Real-time relief map- 1083 ping on arbitrary polygonal surfaces. In Proceedings of the 1084 2005 Symposium on Interactive 3D Graphics and Games. I3D '05; 1085 New York, NY, USA: ACM. ISBN 1-59593-013-2; 2005, p. 1086 155-62. URL: http://doi.acm.org/10.1145/1053427.1053453 1087 doi $10.1145 / 1053427.1053453$

[8] Otaduy M, Jain N, Sud A, Lin M. Haptic display of interaction between 1089 textured models. In IEEE Visualization Conference. 2004, p. 297-304. 1090 doi 10.1109/VISUAL. 2004.37

9] Ren Z, Yeh H, Lin M. Synthesizing contact sounds between textured 1092 models. In Virtual Reality Conference (VR), 2010 IEEE. 2010, p. 139- 1093 46. doi 10.1109/VR.2010.5444799

10] Sterling A, Lin MC. Integrated multimodal interaction using nor- 1095 mal maps. In Proceedings of the 41st Graphics Interface Conference. 1096 GI '15; Toronto, Ont., Canada, Canada: Canadian Information Pro- 1097 cessing Society. ISBN 978-0-9947868-0-7; 2015, p. 33-40. URL: 1098 http://dl.acm.org/citation.cfm?id=2788890.2788898 1099

1] Szirmay-Kalos L, Umenhoffer T. Displacement mapping on the GPU - 1100 State of the Art. Computer Graphics Forum 2008;27(1).

12] Nykl S, Mourning C, Chelberg D. Interactive mesostruc- 1102 tures. In Proceedings of the ACM SIGGRAPH Symposium 1103 on Interactive 3D Graphics and Games. I3D '13; New York, NY, USA: ACM. ISBN 978-1-4503-1956-0; 2013, p. 3744. URL: http://doi.acm.org/10.1145/2448196.2448202 doi $10.1145 / 2448196.2448202$

3] Ho $\mathrm{CH}$, Basdogan C, Srinivasan M. A ray-based haptic rendering technique for displaying shape and texture of $3 \mathrm{~d}$ objects in virtual environments. In ASME Winter Annual Meeting. 1997,

14] Ho CH, Basdogan C, Srinivasan MA. Efficient pointbased rendering techniques for haptic display of virtual objects. Presence: Teleoper Virtual Environ 1999;8(5):47791. URL: http://dx.doi.org/10.1162/105474699566413 doi $10.1162 / 105474699566413$

[15] Theoktisto V, Gonzlez MF, Navazo I. Hybrid rugosity mesostructures (hrms) for fast and accurate rendering of fine haptic detail. CLEI Electron J 2010;:-1.

16] Galoppo N, Tekin S, Otaduy MA, Gross M, Lin MC. Interactive haptic rendering of high-resolution deformable objects. In Proceedings of the 2Nd International Conference on Virtual Reality. ICVR'07; Berlin, Heidelberg: Springer-Verlag. ISBN 978-3-540-73334-8; 2007, p. 215-33. URL: http://dl .acm.org/citation. cfm?id=1770090.1770116

17] Minsky M, Ming Oy, Steele O, Brooks Jr. FP, Behensky M. Feeling and seeing: Issues in force display. $\quad$ SIGGRAPH Comput Graph 1990;24(2):235-41. URL: http://doi.acm.org/10.1145/91394.91451 doi $10.1145 / 91394.91451$

8] Minsky MDRR. Computational haptics: The sandpaper system for synthesizing texture for a force-feedback display. Ph.D. thesis; Cambridge, MA, USA; 1995. Not available from Univ. Microfilms Int.

19] van den Doel K, Pai DK. The sounds of physical shapes. Presence 1996;7:382-95.

[20] O'Brien JF, Shen C, Gatchalian CM. Synthesizing sounds from rigid-body simulations. In Proceedings of the 2002 ACM SIGGRAPH/Eurographics Symposium on Computer Animation. SCA '02; New York, NY, USA: ACM. ISBN 1-58113-573-4; 2002, p. 175-81. URL: http://doi.acm.org/10.1145/545261.545290 doi $10.1145 / 545261.545290$

[21] Raghuvanshi N, Lin MC. Interactive sound synthesis for large scale environments. In Proceedings of the 2006 Symposium on Interactive 3D Graphics and Games. I3D '06; New York, NY, USA: ACM. ISBN 1-59593-295-X; 2006, p. 1018. URL: http://doi.acm.org/10.1145/1111411.1111429 doi 10.1145/1111411.1111429

22] Doel KVD, Kry PG, Pai DK. Foleyautomatic: Physically-based sound effects for interactive simulation and animation. In in Computer Graphics (ACM SIGGRAPH 01 Conference Proceedings. ACM Press; 2001, p. 537-44. 
1104 [23] Hayward V. A brief taxonomy of tactile illusions and demonstrations that can be done in a hardware store. Brain Research Bulletin 2008;75(6):742 -52. URL: http://www.sciencedirect.com/science/article/pii/S0361923008000178 doi http://dx.doi.org/10.1016/j.brainresbull.2008.01.008. special Issue: Robotics and Neuroscience.

[24] Massie TH, Salisbury JK. The phantom haptic interface: A device for probing virtual objects. In Proceedings of the ASME winter annual meeting, symposium on haptic interfaces for virtual environment and teleoperator systems; vol. 55. Chicago, IL; 1994, p. 295-300.

[25] Taylor II RM, Hudson TC, Seeger A, Weber H, Juliano J, Helser AT. Vrpn: a device-independent, network-transparent vr peripheral system. In Proceedings of the ACM symposium on Virtual reality software and technology. ACM; 2001, p. 55-61.

[26] Cook PR, Scavone GP. The synthesis toolkit (stk). In In Proceedings of the International Computer Music Conference. 1999,.

1120 [27] Nakagawa S, Cuthill IC. Effect size, confidence interval and statistical significance: a practical guide for biologists. Biological Reviews 2007;82(4):591-605. URL: http://dx.doi.org/10.1111/j.1469-185X.2007.00027.x doi $10.1111 / \mathrm{j} .1469-185 \mathrm{X} .2007 .00027 . \mathrm{x}$

[28] Otaduy MA, Lin MC. Sensation preserving simplification for haptic rendering. ACM Trans on Graphics (Proc of ACM SIGGRAPH) $2003 ;: 543-$ 53.

[29] Otaduy MA, Lin MC. CLODs: Dual hierarchies for multiresolution collision detection. Eurographics Symposium on Geometry Processing $2003 ;: 94-101$.

[30] Yoon S, Salomon B, Lin MC, Manocha D. Fast collision detection between massive models using dynamic simplification. Eurographics Symposium on Geometry Processing 2004;:136-46.

[31] Otaduy MA, Lin MC. Sensation preserving simplification for haptic rendering. In ACM SIGGRAPH 2005 Courses. SIGGRAPH '05; New York, NY, USA: ACM; 2005,URL: http://doi.acm.org/10.1145/1198555.1198607 doi $10.1145 / 1198555.1198607$

[32] Ren Z, Yeh H, Klatzky R, Lin MC. Auditory perception of geometryinvariant material properties. Proc of IEEE VR 2013;

1141 [33] Ren Z, Yeh H, Lin MC. Example-guided physically-based modal sound $1142 \quad$ synthesis. ACM Trans on Graphics 2013;32(1):Article No. 1. 

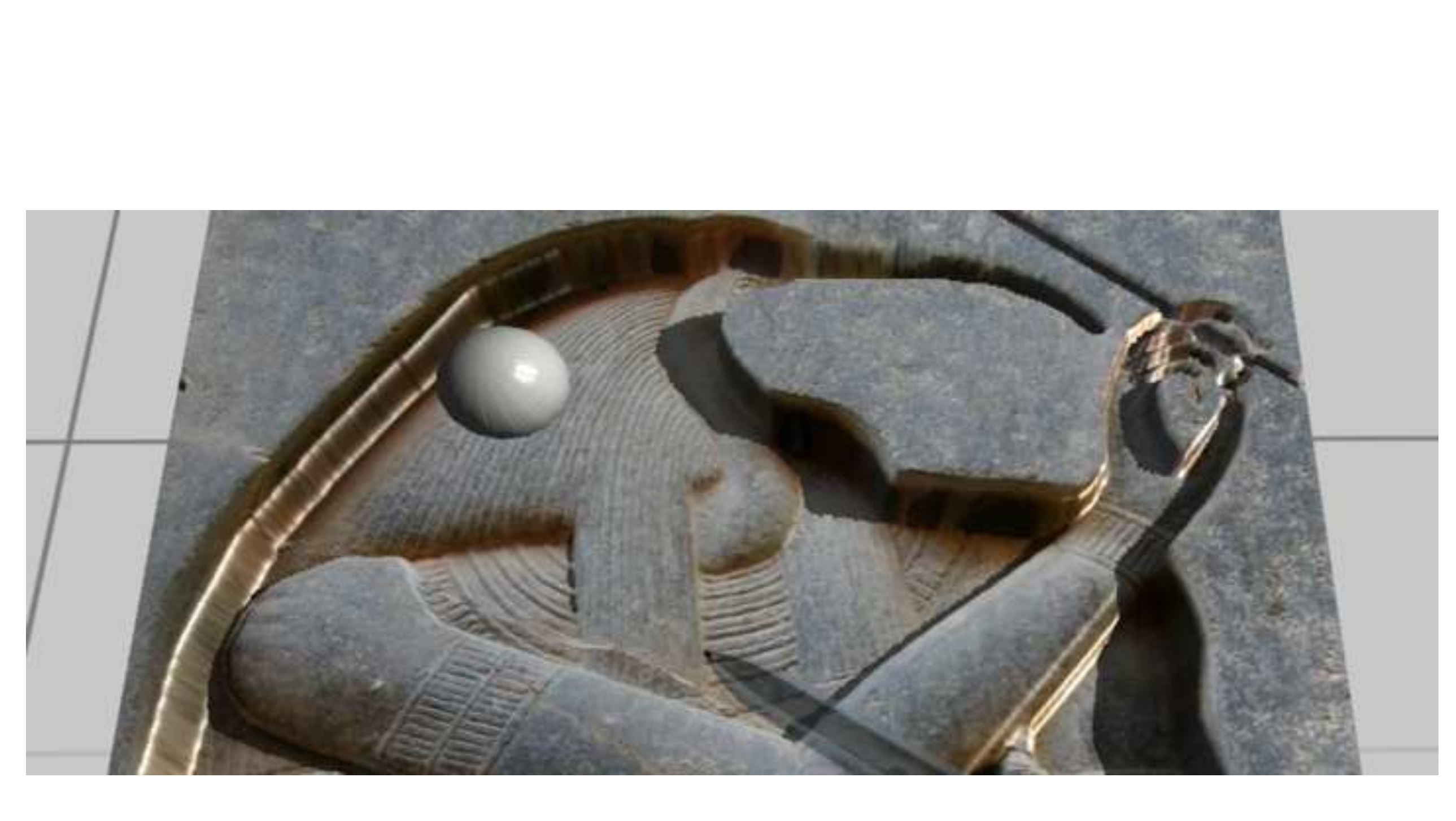